Edith Cowan University

Research Online

ECU Publications 2013

$1-1-2013$

\title{
Correlation of throwing velocity to the results of lower body field tests in male college baseball players
}

\author{
G Lehman \\ Eric J. Drinkwater \\ Edith Cowan University \\ David Behm
}

Follow this and additional works at: https://ro.ecu.edu.au/ecuworks2013

Part of the Sports Sciences Commons

10.1519/JSC.0b013e3182606c79

This is an Author's Accepted Manuscript of: Lehman, G., Drinkwater, E. J., \& Behm, D. (2013). Correlation of throwing velocity to the results of lower body field tests in male college baseball players. Journal of Strength and Conditioning Research, 27(4), 902-908. Available here

This Journal Article is posted at Research Online.

https://ro.ecu.edu.au/ecuworks2013/513 
Running Title: Throwing Velocity Correlations

\title{
Correlation of Throwing Velocity to the Results of Lower Body Field Tests in Male College
}

\section{Baseball Players}

\author{
Authors: Graeme Lehman ${ }^{1}$, Eric J. Drinkwater ${ }^{1,2}$ and David G. Behm ${ }^{1}$ \\ 1. School of Human Kinetics and Recreation \\ Memorial University of Newfoundland \\ St. John’s, Newfoundland, Canada, A1C 5S7 \\ 2. School of Human Movement Studies \\ Charles Sturt University \\ Panorama Avenue, Bathurst, 2795, NSW AUSTRALIA
}

Corresponding Author: Dr. David G. Behm

School of Human Kinetics and Recreation

Memorial University of Newfoundland

St. John’s, Newfoundland, Canada, A1C 5S7

709-864-3408 (tel)

709-864-3979 (fax)

dbehm@mun.ca 
1 Correlation of Throwing Velocity to the Results of Lower Body Field Tests in Male College

\section{Baseball Players}




\section{ABSTRACT}

2 Baseball specific athleticism, potential and performance have been difficult to predict. Increased

3 muscle strength and power can increase throwing velocity but the majority of research has

4 focused on the upper body. The present study sought to determine if bilateral or unilateral lower

5 body field-testing correlates with throwing velocity. Baseball throwing velocity scores were

6 correlated to the following tests; medicine ball scoop toss and squat throw, bilateral and

7 unilateral vertical jumps, single and triple broad jumps, hop and stop in both directions, lateral to

8 medial jumps, 10 and 60 yard sprints, and both left and right single leg 10 yard hop for speed in

942 college baseball players. A multiple regression analysis (forward method), assessing the

10 relationship between shuffle and stretch throwing velocities and lower body field test results

11 determined that right handed throwing velocity from the stretch position were most strongly

12 predicted by lateral to medial jump right (LMJR) and body weight $(B W)\left(R^{2}=0.322\right)$, whereas

13 lateral to medial jump left $(\mathrm{LMJL})\left(\mathrm{R}^{2}=0.688\right)$ predicted left stretch throw. Right-handed

14 shuffle throw was most strongly predicted by LMJR and medicine ball scoop $\left(\mathrm{R}^{2}=0.338\right)$;

15 whereas, LMJL, BW and LMJR all significantly contributed to left-handed shuffle throw

$16 \quad\left(\mathrm{R}^{2}=0.982\right)$. Overall, this study found that lateral to medial jumps were consistently correlated

17 with high throwing velocity in each of the throwing techniques, in both left and right handed

18 throwers. This is the first study to correlate throwing velocity with a unilateral jump in the

19 frontal plane, mimicking the action of the throwing stride.

20 KEY WORDS: jumps, pitching, shuffle, throwing speed, hops, 


\section{INTRODUCTION}

Throwing velocity is an important factor in deciding success in the game of baseball (13).

3 Position players require high throwing velocities to restrict the offense's ability to advance bases

4 and potentially score runs. Pitchers benefit from increased throwing velocity by diminishing the

5 hitter's decision time of whether or not to strike the ball, increasing a pitcher's chance at success

6 (10). High velocity pitches also help set up other pitches such as curve balls or change ups to

7 disrupt the hitters timing.

8 Increasing throwing velocity would benefit any baseball player in a quest to improve

9 their ability to play and to be noticed by coaches and scouts for higher levels of competition.

10 Enhancing throwing mechanics (technique) through proper kinematics and kinetics can optimize

11 the athlete's ability to transfer energy from the ground to upper extremities then ultimately to the

12 ball leading to higher throwing velocity (17). While proper throwing mechanics help maximize

13 performance, research has shown players at youth levels, despite lower throwing velocities, can

14 demonstrate similar mechanics as professional players (22). The difference seen in throwing

15 velocities between these two groups is a result of increased strength and muscle mass (9). This

16 statement is in agreement with DeRenne (3) who stated that throwing velocity could be increased

17 through the improvement in throwing technique or through the use of resistance training (3)

18 stressing the importance of strength to throwing velocity.

19 The implementation of resistance training with the goal of increasing throwing velocity

20 has been successfully studied for many years with the use of several different methods (3).

21 Resistance training in the form of free weight (18), band training (8), medicine balls (16) and 
1 isokinetic machines (27) have all shown positive effects on throwing velocity as well as special

2 resistance training of throwing over-weight and under-weight balls (4). However, there are very

3 few sport specific studies examining the relationship between field tests / exercises and throwing

4 velocity. Furthermore, the majority of the research has focused on the upper body due in part to

5 studies that show the trunk and shoulder generates much of the energy needed to display high

6 throwing velocities (25). Despite the number of studies that focus on upper body strength a

7 survey of Major League Baseball strength and conditioning coaches reported that 15 out of 21

8 respondents believe that a lower body exercise is the most important exercise for the sport of

9 baseball (5). This creates a gap between the research and the application of strength and

10 conditioning practices.

11 Katasumata (11) reported that knee extension maximum voluntary isometric contraction

12 (MVIC) of college aged pitchers correlated highly with throwing velocity however this same

13 relationship was not present in younger pitchers. Spaniol (21) demonstrated higher mean scores

14 in 60 yard dash, horizontal jump, broad jump and throwing velocity with higher levels of

15 competition but no correlation was seen with a lower body test and throwing velocity within any

16 level. The author did however report a significant relationship between throwing velocity and

17 grip strength. These few correlational studies used similar bilateral movements whereas the

18 baseball throw emphasizes distinct or separate functions for each leg. In accordance with the

19 concept of training specificity (28), research is necessary to help athletes and coaches incorporate

20 field tests that would correlate highly with throwing velocity. 
This lack of a correlation between lower body strength and throwing velocity is

2 perplexing due to some research that demonstrates that increased lower body force production

3 during the act of throwing allow for higher throwing velocities. MacWilliams et al. (14)

4 demonstrated that increases in force production of the trail leg in the direction of the intended

5 target in the frontal plane correlated with higher throwing velocity leading the authors to suggest

6 that this allowed for more potential energy to be transferred to the ball. The strength of the lead

7 leg was identified as a difference between high and low velocity throwing groups by Matsuo (15)

8 who reported that the ability to demonstrate knee extension upon landing was a common

9 characteristic among high velocity throwers. Members of the slow throwing velocity group

10 continued further into knee flexion. The authors concluded that the lead leg provides both a

11 stable base while also redirecting energy superiorly towards the upper extremities. This is

12 congruent with Pappas et al. (17) description of throwing as a sequential activation of body parts

13 through a link segment beginning with the contralateral foot progressing through the trunk to a

14 rapidly accelerating upper extremity

15 The act of throwing while bilateral in nature requires different actions during the

16 throwing cycle from both lower extremities. The trail leg performs a concentric action (14) in

17 the frontal plane while the lead leg eccentrically absorbs the energy created by the trail leg then

18 concentrically redirects kinetic energy up the kinetic chain via a concentric contraction (15). The

19 difference between the lower extremities was noted by Tippett et al. (24) who reported

20 differences in strength and range of motion in the lower extremities of college baseball pitchers.

21 This study did not however correlate any of their findings with throwing velocity. Other studies 
1 have exclusively used bilateral lower body movements in an attempt to correlate with throwing

2 velocity (20) with the exception of running which is a cyclical action unlike throwing. Based on

3 the research that describes the dynamic and independent actions of the lower extremities one can

4 hypothesize that tests like isometric contractions, maximum strength, bilateral movements or

5 actions in sagittal plane would correlate poorly with throwing velocity.

6 There is no research examining frontal, unilateral and non-lab based tests to predict

7 throwing velocity. Thus the purpose of this study was to determine which lower extremity field

8 tests correlate with throwing velocity in order to provide coaches and athletes with more

9 direction in creating training programs that are highly associated with increases in throwing

10 velocity. In order to achieve this objective, lower body field tests, which include bilateral and

11 unilateral actions along with movements in various planes and muscle contractions (eccentric

12 and concentric) were correlated to throwing velocity results. According to the concept of training

13 specificity (28), field tests, which most closely simulate the throwing action should more

14 efficiently train those muscles associated with a high throwing velocity.

\section{METHODS}

16 Experimental Approach to the Problem

17 This study was designed to determine if the chosen bilateral and unilateral lower body

18 field tests were correlated to throwing velocity. The experimental protocol was conducted

19 during the fall season of the college baseball season, which primarily consists of practices and

20 intersquad games. Individual multiple regression analyses (forward method) were calculated 
1 between both shuffle and stretch throwing velocities of left and right handed players (dependent

2 variable) and the results of the lower body field tests (independent variables). The lower body

3 field tests consisted of medicine ball scoop toss, medicine ball squat throw, bilateral vertical

4 jump, left leg vertical jump, right leg vertical jump, broad jump, triple broad jump, hop and stop

5 from left to right, hop and stop from right to left, lateral to medial jump right, lateral medial jump

6 left, 10 yard sprint, 60 yard sprint and both left and right single leg 10 yard hop for speed. To

7 determine which exercises performed on a frontal or sagittal plane with unilateral or bilateral

8 actions provided the greatest correlation with throwing velocity, a variety of field tests were

9 conducted.

10 Subjects

11 Forty-two college level baseball players from two teams (Northwest Athletic Association

12 of Community Colleges $(n=19)$; National Association of Intercollegiate Athletics $(n=23)$ were

13 used for this study, all of who had at least 10 years of experience playing baseball and at least 2

14 years experience with resistance training. The mean age was 19.8 years $(+/-1.2)$. The subjects

15 had a mean height and weight of $183.3 \mathrm{~cm} .(+/-9)$ and $83.1 \mathrm{~kg}(+/-14)$ respectively with

16 throwing velocities ranging from $74-87$ miles per hour $(118-141 \mathrm{~km} / \mathrm{hr})$. Each subject had not

17 reported any arm problems within the last 3 months. Participants were verbally informed of the

18 procedures and read and voluntarily signed a consent form and a Physical Activity Readiness

19 Questionnaire (PAR-Q) before participation (23). The Memorial University of Newfoundland

20 Human Investigation Committee approved the study.

21 Testing Schedule 
The subjects were carefully familiarized with the testing protocols 3 weeks in advance of

2 the actual testing date in order to minimize the learning effect. After a standardized 10-minute

3 warm-up period that included low-intensity running, dynamic mobility drills and several

4 acceleration runs, subjects were randomly assigned to one of four testing stations. Physical field

5 tests were divided into four groups: (1) medicine ball throws (2) vertical jumps (3) horizontal

6 jumps and (4) sprints and timed hops.

$7 \quad$ Medicine Ball Throws

8 Two types of medicine ball throws (squat and scoop) were performed on the field and

9 consisted of three throws with the farthest throw being recorded. A 2.7kb (6lbs) medicine ball

10 was used for all of the tests. One investigator marked the spot where the ball landed while

11 another would measure the distance from the starting line to the landing spot. Each subject

12 performed three throws with the farthest throw being recorded. Thirty seconds of recovery were

13 allocated between throwing attempts to prevent muscular fatigue.

14 For the medicine ball squat throws, subjects were instructed to perform a

15 countermovement (flexion and extension) with the lower body and explosively extend through

16 the hips and knees into a forward jump while performing a chest pass motion with both arms

17 extending to allow for maximal power. When performing, medicine ball scoop throws subjects

18 stood facing away with their backs towards the intended target. Subjects were instructed to grasp

19 the medicine ball with both hands and swing the ball between their legs before explosively

20 extending their hips and throwing the ball as far as possible behind themselves.

21 Vertical Jumps 
The bilateral and unilateral vertical jumps tests were recorded using a contact mat (Jump

2 Mat, Axon, USA). For the bilateral jump, subjects were asked to perform a maximal jump on the

3 contact mat from a stationary position while standing on both feet. Subject's performed a

4 preparatory countermovement with the lower body coupled with arm swings to achieve maximal

5 height. Arm swings were allowed since subjects were accustomed to jumping with an arm

6 swing action. The jumping height was calculated from the flight time. Each subject performed

7 three jumps with approximately 10 seconds between jumping attempts. Subjects were instructed

8 not to tuck their legs upon landing in an attempt to increase flight time. The best reading was

9 used for further analysis.

10 When performing unilateral jumps, subjects were asked to perform a maximal jump on

11 the contact mat from a stationary position while standing only on one foot. Subjects performed a

12 preparatory countermovement with the lower body coupled with dual arm swing to achieve

13 maximal height. Subjects performed a one legged take off and were instructed to land on both

14 feet simultaneously. The jumping height was calculated from the flight time. Each subject

15 performed three jumps with approximately 10 seconds between jumping attempts. The best

16 reading was used for further analysis. Following a 90 second recovery, subjects repeated this

17 process on the opposite leg. The order was randomized.

18 Horizontal Jumps

19 A series of horizontal jumps were performed in the same order. Approximately 10

20 seconds rest was given between attempts on each test and 3 minutes were given between

21 different horizontal jump tests. The horizontal broad jump was performed on turf (both takeoff 
1 and landing) from a stationary position, with arm swings, a 2 foot take-off and was measured

2 with a tape measure. Each subject performed two maximal jumps; the distance was measured

3 from the heel of the foot closest to the starting line. The best of the three jumps were recorded for

4 further analysis. For the hop and stop, subjects stood at the starting line on one foot and were

5 instructed to perform a countermovement forward jump along with dual arm swing to allow for

6 maximal distance. Subjects were required to land on their opposite leg and come to a complete

7 stop with no trunk or limb movement in less than one second. Subjects were allotted five

8 attempts to land three jumps that met the above criteria the farthest of which was recorded for

9 further analysis. If three scoring jumps were not accomplished subjects were allotted 120

10 seconds of rest before attempting again. Distance was measured from the back of the heel to the

11 starting line. One investigator determined if the jump counted by starting a stop watch upon

12 landing and stopping it upon the cessation of movement. Subjects then repeated the process

13 jumping with the opposite leg. The order of the jumps was randomized.

14 Lateral to medial jump (LMJ)

15 Subjects were instructed to stand parallel to the starting line on their left foot with the

16 inside of their foot closest to the starting line. Subjects were instructed to perform a

17 countermovement with their lower body and jump as far as possible to their right in the frontal

18 plane while landing on both feet simultaneously parallel to the starting line. The distance was

19 recorded from the outside of the left foot to the starting line. Three attempts were given with

20 approximately 10 seconds of rest; the greatest distance was recorded for further use. This

21 process was repeated on the opposite leg. 
1 Bilateral Triple Jump

2 Three consecutive two-legged hops were recorded with the use of a measuring tape fixed

3 to the ground perpendicular to the starting line. Participants stood with the great toe of both feet

4 at the starting line. They performed 3 consecutive maximal hops forward with minimal time

5 spent on the ground to allow for maximal use of stored elastic energy. Arm swings were

6 allowed. The investigator measured the distance from the starting line to the point where the

7 heel of the foot closest to the starting line landed upon completing the third hop. Three trials

8 were given with the greatest being recorded for further use.

$9 \quad$ Speed Tests

10 All speed tests were conducted on an Astroturf field and were recorded with an infrared

11 testing device (Speed Trap II; Brower Timing Systems, Draper, UT, USA). For the 10 yard

$12(9.14 \mathrm{~m})$ sprint, subjects stood in a two-point stance with one foot just behind the starting line.

13 Subjects performed two attempts with approximately 120 seconds of rest between attempts with

14 the fastest of the three attempts recorded for further use. The 60-yard (54.86m) sprint (traditional

15 baseball test) was completed by having subjects stand in a two-point stance with one foot just

16 behind the starting line. Subjects performed two attempts with approximately 120 seconds of

17 rest between attempts with the faster of the two attempts recorded for further use. With the 10-

18 yard (9.14m) single leg hop test, subjects stood on one leg just being the starting line and

19 covered the 10 yard distance as fast as possible while hopping exclusively on the same leg. Two

20 attempts were given with approximately 120 seconds of rest between attempts with the faster of 
1 the two being recorded for further use. Following a three-minute recovery, this process was

2 repeated for the opposite leg. Choice of legs was randomized.

3 Throwing velocity

After an adequate throwing warm up, each subject was given 3 attempts to reach their

5 maximal throwing velocity. Each subject threw overhand from flat ground at maximal effort to a

6 target positioned at approximately chest level from 18.44m away, which is the distance between

7 the pitching rubber and home plate. Throwing velocity was recorded from a calibrated Jugs

8 Sport Radar gun (Jugs Pitching Machine Company, Tualatin, OR, USA) as the ball left the

9 player's hand and is accurate within $0.22 \mathrm{~m} / \mathrm{s}$.

10 Stretch Throwing Velocity

11 Athletes started with both feet together and were allowed to take one stride towards the

12 target. This mimics the "stretch" position that pitchers are forced to throw from when runners are

13 on base. Thirty seconds were given between throwing attempts to prevent muscular fatigue. The

14 throw with the highest velocity was recorded.

15 Shuffle Throwing Velocity

16 Following the 3 throws from the stretch position each athlete performed an additional 3

17 throws where they were allowed to build momentum by shuffling in the frontal plane towards the

18 target within a 3-meter ( 10ft) limit. Again subjects threw overhand from flat ground at maximal

19 effort to a target positioned at approximately chest level from $18.44 \mathrm{~m}$ away. Thirty seconds

20 were given between throwing attempts to prevent muscular fatigue. The throw with the highest

21 velocity was recorded. 


\section{RESULTS}

\section{Stretch - Right Hand Throw}

\section{Statistical Analysis}

\section{Equation 1}

The mean and SD of the selected anthropometric and physical performance tests were calculated for both left and right handed throwing subjects (Tables 1 \& 2). Four separate multiple regression analyses were performed (forward method) to determine the contribution of anthropometric as well as all physical capability tests (independent variables) to throwing velocity scores (dependent variable) with a shuffle approach and from the stretch position. This was performed for both right handed $(n=33)$ and left handed $(n=9)$ throwers. Statistical Analysis was performed with PASW Statistics 17 (Release Version 17.0.2, SPSS Inc., Chicago, Illinois, USA). Results are expressed with the adjusted $\mathrm{R}^{2}$, and regression equations with the standard error of the estimate (SEE) for each regression.

Equation 1 represents the results of the regression analyses between right handed throwing velocity from the stretch position. The scores from both the anthropometric and physical performance tests showed that 2 factors, lateral to medial jump right (LMJR) and body weight (BW) played substantial contributing roles throwing velocity (adjusted $\mathrm{R}^{2}=0.322$, $\mathrm{F}=8.609, \mathrm{SEE}=6.437, p=0.001)$. These results indicated that approximately $32.2 \%$ of the variance of ball throwing velocity from the stretch position in right-handed throwers can be accounted for by the LMJR scores and BW.

$$
\mathrm{y}^{\wedge^{\prime}=101.9+(\mathrm{LMJR} \times-0.050)+(\mathrm{BW} \times 0.374)}
$$




\section{Adjusted R2=0.322, SEE=5.77}

13 Stretch - Left Hand Throw

\section{Shuffle - Right Hand Throw}

\section{Equation 2} LMJL scores and BW.

Equation 2 represents the results of the regression analyses between right handed throwing velocities with a shuffle approach. Regression scores from both the anthropometric and physical performance tests showed that 2 factors, lateral to medial jump right (LMJR) and medicine ball scoop (MB Scoop) played substantial contributing roles throwing velocity (adjusted $\mathrm{R}^{2}=0.338, \mathrm{~F}=9.181, \mathrm{SEE}=6.795, p=0.001$ ). These results indicated that approximately $33.8 \%$ of the variance of ball throwing velocity from the stretch position in righthanded throwers can be accounted for by the LMJR and MB Scoop scores.

$$
\mathrm{y}^{\wedge^{\prime}=101.2+(\text { LMJR } \times-0.068)+(\text { MB Scoop } \times 0.021)}
$$

$$
\text { Adjusted R2=0.338, SEE }=6.80
$$

Equation 3 represents the results of the regression analyses between left handed $(n=9)$ throwing velocity from the stretch position and the scores from both the anthropometric and physical performance tests showed that only one factor, lateral to medial jump left (LMJL) played substantial contributing role throwing velocity (adjusted $\mathrm{R}^{2}=0.688, \mathrm{~F}=18.659$, SEE $=3.786, p=0.003)$. These results indicated that approximately $68.8 \%$ of the variance of ball throwing velocity from the stretch position in left-handed throwers can be accounted for by the 
1

3 Shuffle - Left Hand Throw

\section{Discussion \\ Discussion}

\section{Equation 4}

3

$\mathrm{y}^{\wedge^{\prime}=135.4+(\operatorname{LMJL} \times-0.092)}$

Adjusted R2=0.688, SEE=3.79

Equation 4 represents the results of the regression analyses between left handed (n=9)

throwing velocity with a shuffle approach. These scores from both the anthropometric and physical performance tests showed that 3 factors, LMJL, BW and LMJR played substantial contributing roles throwing velocity (adjusted $\mathrm{R}^{2}=0.982, \mathrm{~F}=144.115$, $\mathrm{SEE}=.648, p=0.001$ ).

These results indicated that approximately $98 \%$ of the variance of ball throwing velocity from the stretch position in left-handed throwers can be accounted for by the LMJL, BW and LMJR.

$$
\mathrm{y}^{\wedge^{\prime}=208.0+(\text { LMJL } \times-0.072)+(B W \times-0.770)+(\text { LMJR } \times-0.206)}
$$

$$
\text { Adjusted R2 }=0.982 \text {, SEE }=0.65
$$

There was a consistent appearance of the lateral to medial jumps as a factor correlated to high throwing velocity in each of the throwing techniques for both left and right handed throwers. This was the first published study to correlate throwing velocity to a unilateral jump in the frontal plane, which mimics the action of the stride.

19 The importance of the stride was noted in a biomechanical study of the throwing motion by Stodden et al. (22) who reported that the stride functions as the initial factor to generate and 
transfer force of momentum up through the kinetic chain by initiating linear momentum of the

2 body towards the intended target. This need for linear velocity has been reported with other

3 throwing activities. Top level javelin throwers exhibited both longer strides and higher

4 approach velocities (1) while Salter et al. (19) demonstrated that $87.5 \%$ of ball release speed for

5 a cricket bowler can be attributed to run-up velocity, angular velocity of the bowling arm,

6 vertical velocity of the non-bowling arm, and stride length.

$7 \quad$ This correlation between lateral to medial jump scores and throwing velocity is congruent

8 with the information provided by MacWilliams et al. (14) which stated increased ground reaction

9 forces created by the trail leg in the direction towards the target were highly correlated with ball

10 velocity. Theoretically, the increase in momentum would allow baseball players to transfer more

11 energy through the kinetic chain from the trunk, to the throwing arm, and finally to the ball to

12 produce increased ball velocities. While the ability to generate momentum is important, one must

13 be careful to not artificially produce linear momentum towards the intended target.

14 MacWilliams (14) noted that while the correlation of ground reaction force to throwing velocity

15 was high $\left(r^{2}=0.82\right)$ some subjects demonstrated the reverse trend with what the authors called

16 “overthrowing”. The authors noted that the athletes must integrate the powerful leg drive as a

17 natural part of their throwing motion due to its complexity. If peak ground reaction forces occur

18 too early during the throwing motion, throwing velocity is reduced (6). MacWilliams et al. (14)

19 found that the forces were gradually built up and peaked just prior to the lead foot making

20 contact with the ground. The need to create momentum towards the target is taught by some 
1 pitching coaches who stress the involvement of the lower body by emphasising the need to

2 "push" or "drive“ towards the target as part of a well-integrated pitching motion (7).

3 The specificity of the lateral to medial horizontal jump may be the primary reason that it 4 correlated to high throwing velocity. Strength and conditioning coaches apply the principal of 5 specificity to athletes who desire the ability to improve a specific task. The specificity principal 6 implies that to become better at a particular skill the training must involve the skill by replicating 7 the biomechanical movements (28). Traditional bilateral tests such as vertical, horizontal 8 jumping and running speed in the sagittal plane did not substantially correlate to high throwing 9 velocity in the current study. These results agree with the findings of Spaniol (20) who did not 10 find any correlation between either running speed (60 yard dash) or lower body power (vertical 11 jump) and throwing velocity.

12 The correlation between throwing velocity and lateral to medial jumps suggest that there 13 is a high degree of specificity in regards to power in a specific direction and plane of movement.

14 The poor carryover from training in one plane of motion and testing in another has been shown

15 by King and Cipriani (12) who reported reduced improvements in vertical jump scores of 16 subjects that trained exclusively with frontal plane plyometric exercises compared to those that 17 trained in the sagittal plane. Young et al. (28) also found low transferability between linear speed 18 and agility.

The results of this study also demonstrated that body weight had a substantial relationship

20 with throwing velocity for right handed throwers from the stretch position and left handed

21 throwers with a shuffle approach. These findings are congruent with those from Werner et al. 
1 (26). Increased body weight increases the total amount of energy that can be ultimately

2 transferred to the ball allowing for higher throwing velocity. In each case that body weight was a

3 substantial factor it was also coupled with the lateral to medial jump which indicates increased

4 amounts of body mass must be accompanied by the appropriate amounts of power. Added body

5 mass in the form of fat would not be beneficial as it can be assumed that it would decrease the

6 lateral to medial jump scores. Increased distance from a lateral to medial jump coupled with

7 increased body weight would again account for increased amounts of kinetic energy in the

8 direction of the target allowing for high throwing velocity scores.

$9 \quad$ Throwing a baseball with high velocity requires a complex combination of kinematics

10 and kinetics that must be in place in order to optimize the athlete's ability to transfer energy to

11 the baseball. However if these motor patterns are in place due to years of practice the results of

12 this study lead us to believe that increased levels of power in the frontal plane have a high

13 relationship with higher throwing velocity scores. Future studies will have to determine if

14 increases in the athlete's ability to jump further in the frontal plane will translate into higher

15 levels of throwing velocity.

16 PRACTICAL APPLICATIONS

17 This study found that lateral to medial jumps, which measured the athlete's ability to

18 create power in the frontal plane, which is specific to the act of throwing a baseball, best

19 predicted throwing velocity. Coaches should integrate unilateral jumping drills and resistance

20 training in the frontal plane in order to apply the principal of specificity. Traditional exercises 
1 performed in the sagittal plane (lunges, single leg squats, deadlifts) should not be excluded but

2 rather serve as a means of increasing overall lower body power in the initial phases, such as

3 anatomical adaptation, hypertrophy and maximum strength of an off-season strength program

4 (2). The de-emphasis of frontal plane movements following the baseball season which consists

5 primarily of frontal and transverse plane movement like throwing and hitting will serve both as

6 change of stimulus while potentially reducing the chance of an overuse injury.

7 It is our opinion that frontal plane unilateral exercises would be best suited during the

8 final phases of a periodized program when strength is converted to power following a well-

9 planned periodized program. (2). Traditionally this final phase would consist of sagittal plane

10 movements like vertical jump, depth jumps or medicine ball squat throws however the results of

11 this study indicate that plane specific movements would best suit the baseball athlete who wishes

12 to increase throwing velocity. 


\section{REFERENCES}

1. Bartlelt, R, Muller, E, Lindinger, S, Brunner, F, and Morris, C. Three-dimensional evaluation of the kinematic release parameters for javelin throwers of different skill levels. $J$ of App Biomech 12: 58-71, 1996.

2. Bompa, TO. Periodization Training for Sports. Human Kinetics. Champaign, Il, 1999.

3. DeRenne, C, Ho, KW, and Murphy, JC. Effects of general, special and specific resistance training on throwing velocity in baseball: a brief review. J Strength Cond Res 15(1): 148-156, 2001.

4. DeRenne, C, Buxton, BP, Hetzler, RK, and Ho, KW. Effects of under-and over-weighted implement training on pitching velocity. J Strength Cond Res 8: 247-250, 1994.

5. Ebben, WP, Hintz, MJ, and Simenz CJ. Strength and conditioning practices of Major League Baseball strength and conditioning coaches. J Strength Cond Res 19(3): 538-546, 2005.

6. Elliot, B, Grove, R, and Gibson, B. Timing of the lower limb drive and throwing limb movement in baseball pitching. Int J Sports Biomech 4: 59-67, 1988.

7. Empey, D. Developing Pitchers. Vancouver, BC: Club House Publishing, 2002.

8. Escamilla, RF, Fleisig, GS, Yamashiro, K, Mikla, T, Dunning, R, Paulos, L, and Andrews, JR. Effects of a 4-week youth baseball conditioning program on throwing velocity. $J$ Strength Cond Res 24(12): 3247-3254, 2010.

9. Fleisig, G, Barrentine, S, Zheng, N, Escamilla, R, and Andrews, JR. Kinematic and kinetic comparison of baseball pitching among various levels of development. J Biomech 32: 13711375, 1999.

10. Hay, JG. Baseball. In: Biomechanics of Sports Technique (3rd ed.). Englewood Cliffs, NJ: Prentice-Hall, Inc., 1985. pp. 188-213. 
11. Katsumata, Y, Kawakami, Y, and Fukunaga T. Relationships Between Throwing Velocity and

12. King, JA and Cipriani, DJ. Comparing preseason frontal and sagittal plane plyometric programs on vertical jump height in high-school basketball players. J Strength Cond Res 6 24(8): 2109-2114, 2010.

13. Kohmura, Y, Aoki, K, Yoshigi, H, Sakuraba, K, and Yanagiya, T. Development of a baseballspecific battery of tests and a testing protocol for college baseball players. J Strength Cond

14. MacWilliams, B, Choi, T, Perezous, M, Chao, E, and McFarland, E. Characteristic groundreaction forces in baseball pitches. Am J Sports Med 26: 66-71, 1998.

15. Matsuo, T, Escamilla, R, Fleisig, G, Barrentine, S, and Andrews, J. Comparison of kinematic and temporal parameters between different pitch velocity groups. J Appl Biomech 17: 1-13, 2001.

16. Newton, RU, and McEvoy, KP. Baseball throwing velocity: A comparison of medicine ball training and weight training. J Strength Cond Res 8: 198-203, 1994.

17. Pappas, AM, Zawacki, RM, and Sullivan, TJ. Biomechanics of baseball pitching. A preliminary report. Am J Sports Med 13: 216-222, 1985.

18. Popescue, MG. Weight training and the velocity of a baseball. Athlet J 55: 74-106, 1975.

19. Salter, CW, Sinclair, PJ, and Portus, MR. The associations between fast bowling technique and ball release speed: A pilot study of the within-bowler and between-bowler approaches. $J$ Sports Sci 25(11): 1279 - 1285, 2007.

20. Spaniol, FJ. Predicting throwing velocity in college baseball players. J Strength Cond Res 11: 286, 1997.

21. Spaniol, FJ. Baseball athletic test: a baseball-specific test battery. J Strength Cond Res 31: 26-29, 2009. 
2 22. Stodden, DF, Langeddorfer, SJ, and Fleisig, GS. Kinematic constraints associated with the 3 acquisition of overarm throwing part I: Step and trunk actions. Res Quar for Exer and Sport 4 77: 417-427, 2006.

23. Thomas, S, Reading, J and Shephard, RJ. Revision of the Physical Readiness Questionnaire (PAR-Q). Can J Sport Sci 17(4): 338-345, 1992.

24. Tippett, SR. Lower extremity strength and active range of motion in college baseball pitchers: A comparison between stance leg and kick leg. J Orthop Sports Phys Ther 8: 10-14, 1986.

25. Toyoshima, S, Hoshikawa, T, and Miyashita, M. Contributions of the body parts to throwing performance. In: Biomechanics IV. R.C. Nelson and C.A. Morehouse, eds. Baltimore: University Park Press, 1974. pp. 169-174.

26. Werner, SL, Suri, M, Guido Jr, JA, Meister, K, and Jones, DG. Relationships between ball velocity and throwing mechanics in collegiate baseball pitchers. J Shoulder Elbow Surg 17(6): 905-908, 2008.

27. Wooden, MJ, Greenfield, B, Johanson, M, Litzelman, L, Mundrane, M, and Donatelli, RA. Effects of strength training on throwing velocity and shoulder muscle performance in teenage baseball players. J Orthop Sports Phys Ther 15: 223-228, 1992.

28. Young, WB, McDowell, MH, and Scarlett, BJ. Specificity of sprint and agility training methods. J Strength Cond Res 15: 315-319, 2001. 